\title{
Reflexiones onomasiológicas en torno al papel de la administración en los programas de Español como lengua extranjera 1
}

\author{
Bianchinetta Benavides Segura2 \\ Gisselle Herrera Moreral \\ Universidad Nacional. Costa Rica
}

\begin{abstract}
REsUMEN
Es un planteo de base teórica sobre la incorporación efectiva de la conciencia intercultural en los programas de Español como lengua extranjera (ELE) y de la influencia de las personas encargadas de administrar y facilitar su adecuada inclusión. La competencia en una lengua extranjera supone tanto capacidades lingüísticas, como el conocimiento y apropiación del conjunto de valores, creencias y normas culturales que conforman la identidad individual y colectiva de una comunidad académica.
\end{abstract}

\begin{abstract}
ABSTRAer
This is a theoretically-based proposal conceming the effective incorpora- tion of intercultural awareness in Spanish as a Foreign Language (SFL) pro- grams and the influence of administering facilitating its adequate inclusion. Proficiency in a forei gn language involves not only linguistic skills but also a knowledge and appropriation of the cultural values, beliefs and norms integrated in the individual and collective identity of an academic community.

Palabras clave: español como lengua extranjera, comunicación i ntercultural, bilingüismo

Keywords: Spanish as a foreign language, intercultural communication, bilingualism
\end{abstract}

Recibido: 6 de junio de 2009; aceptado: 20 de febrero de 2010.

2 Dcuela de Literatura y Ciencias del Lenguaje. Correo electrónico: bbenavid@ una.ac.cr

3 Dcuela de Literatura y Ciencias del Lenguaje. Correo electrónico: gherrer@una.ac.cr 
La onomasiología invita a la investigación en torno a los significantes de un concepto y al punto de vista que los usuarios adoptan en relación con esos significantes4• El presente artículo surge de la experiencia de sus autoras acerca del efecto del punto de vista de los administradores de los programas de Español como lengua ex- tranjera (ELE) en este tipo de currículo. Además, plantea algunas consideraciones teóricas acerca de la reflexión sobre los significan- tes subyacentes en la comunicación transaccional e intercultural en dichos programas.

La finalidad de la adquisición de una lengua es, principalmen- te , la comunicación transaccional efectiva; una negociación de sig- nificado y contexto que surge como resultado de las interacciones entre la experiencia y el conocimiento del mundo que nos rodea, de nuestras expectativas, de nuestro dominio del tema. Sin embargo, cuando nos comunicamos ya en el ámbito internacional, lo hacemos interculturalmente. En esta dimensión , llegar a tener una competen- cia en una lengua extranjera requiere no solo de las capacidades lingüística, pragmática y sociolingüística, sino que obliga, además , a identificar aquellos valores y normas culturales que definen nuestra identidad individual (como actores del proceso de enseñanza-aprendizaje) y colectiva (como miembros de una comunidad académica intercultural y responsable) .

Desde hace poco más de diez años, el auge acelerado de los programas de "español para extranjeros» ha mostrado la necesidad de contar con una visión clara de lo que supone este tipo de currículo, necesidad que consideramos inicia con el análisis de la etimología misma del significante extranjeros. Para la Real Academia Española, la etimología de los conceptos puede producir una interpretación espontánea dada vulgarmente a una palabra cuando la relacionamos con otra de distinto origen; una relación que, por la frecuencia de

4 Real Academia de la Lengua Española (RAE). Diccionario de la lengua española. Vigésima pñ· mera edición. (Madñd: Espasa Calpe, 1992) 1478. 
su Uso, puede originar cambios o deformaciones semánticas5 - En el contexto de los programas de ELE, un primer acercamiento etimológico muestra que el significante extranjeros converge semánticamente con el significado dado a ese mismo serna dentro del contexto del turismo. Según nuestra experiencia, quienes administran estos programas suelen limitarlos a una simple venta de servicios, cuasiturísticos en muchos casos, dejando de lado criterios académicos vitales para el crecimiento curricular, como lo son la investigación y promoción de las competencias interculturales de los académicos y de los estudiantes.

El primer aspecto fue constatar que, para la mayoría de los participantes (administradores, profesores e incluso los mismos estudiantes), la actividad académica se veía opacada frente a la oferta de servicios: administración del hospedaje, uso del transporte , fo- mento de excursiones, generación de fondos, entre otros. En escasas ocasiones se evidenciaba ese componente pedagógico tan intrínseco del aprendizaje de una lengua extranjera: la dimensión intercultural . Esta situación fue producto de una batalla ideológica entre lo que se considera y lo que debería considerarse como parte de un programa de ELE.

Como resultado, podemos señalar cinco áreas que, lejos de contar con un acercamiento onomasiológico que delimite la comunicación intercultural efectiva, más bien se han orientado a un enfoque etimológico, muchas veces ideológico y, casi siempre, poco intercultural. Tales áreas son6: el entendimiento real de la relación lengua y cultura; la comprensión cultural profunda sobre los valores y actitudes de los participantes; la oportuna visualización de la utilidad del aprendizaje de una lengua extranjera desde una perspectiva intercultural; la selección profesional de metodologías y materiales didácticos; y la profesionalización de la competencia intercultural de los actores del proceso pedagógico. La experiencia demuestra que

\footnotetext{
5 目, 924.

6 El orden de aparición no disminuye su importancia.
} 
los administradores son , en primera instancia, quienes han obviado esas cinco áreas del eje curricular. A continuación se analiza cada una de ellas , y al mismo tiempo nos detendremos a reflexionar sobre algunos aspectos importantes.

Coftpreftsióft de la relacióft efttre leftgua y cultura

Quien aprende una lengua extranjera no alcanza adquirir una competencia comunicativa en la lengua meta sin que haya interiorizado los patrones y valores de los hablantes de esa cultura. Los conceptos de lenguaje y sociedad no han de separarse y éstos no deben contemplarse sin ignorar la comunicación verbal y la no verbal del ser humano. Al respecto, surgen las preguntas: ¿cuáles hablantes?; ¿cuál cultura?; ¿cuáles patrones?; ¿cuáles valores?; ¿cuál identidad?

1. ¿ Cuáles hablantes? En nuestro caso, serían los hablantes de las variedades empleadas en Costa Rica o, si queremos ampliar geográficamente el alcance de esta reflexión , las hispanoamericanas. No se puede seguir con la práctica de enseñar la variedad peninsular $y$, mucho menos, que sean los docentes mismos quienes fomenten su uso, amparados bajo una premisa falsa de que es la variedad más útil o de que todos los estudiantes i rán a España algún día, olvidando a los millones de hispanoamericanos en todo el mundo.

2. ¿ Cuál cultura? Es un concepto que varía de autor a autor o de disciplina a disciplina; no obstante, las definiciones brindadas por Plog y Bates en Roldán7, Y Taylor en Prado y otros8, son afines. En Plog y Bates se define como el sistema de creencias, valores, costumbres, conductas y artefactos compartidos, que los miembros de una sociedad usan en interacción entre ellos mismos y con su

7 Antonio Roldán Tapia, «Multiculturalidad e interculturalidad», consultado 1 de setiembre 201 1 ,

<http://www.fongdcam.org/manuales/educacionintercultural/datos/docs/ArticulyDocu mentosI

Clarificacion\%20de\%20c0nceptos/cuIMultIntr/MULTICULTURALIDAD\%20EINTGERC ULTU- RALIDAD\%20T9.pdf>. 
8 Josefina Prado Aragonés y otros, La Galaxia Digital. Lenguaje y cultura sinfronteras en la información (Andalucía: Grupo Editorial Universitario, 2(03).

era de 
IIIII IIdo, y que son transmitidos de generación en generación a través IIl'l aprendizaje. Por lo tanto, la cultura que se adquirirá en nu.estro pa ís será la costarricense. Al no ser un producto único e inmutable, si 110 cambiante y dinámico en función de la interacción social y con II tras culturas, quienes administran su enseñanza, en nuestro país, no plleden obviar la influencia de las culturas de entorno (por ejemplo: la nicaragüense, la colombiana, la mejicana, la estadounidense, entre otras) así como la propia del aprendiente.

3. ¿ Cuáles patrones? Nos enfrentamos a la necesidad de diferenciar entre dos tipos de patrones: el primero, reconocer la diferencia entre dos culturas9: la cultura superficial, referida a los detalles que dan cuenta de la forma de vida diaria y la cultura profunda, que incluye los resultados artísticos, los logros intelectuales, los hechos heroicos, los conceptos elevados de nobleza espiritual, las diferentes formas de expresión significativa del pensamiento y el estilo étnico. Con respecto a cuál enseñar, ambas son indispensables. No obstante, la profunda requiere más cuidado porque es la que no se percibe a simple vista y necesita mayor tiempo para su comprensión. Muchos administradores insisten en que los docentes acompañen a sus estudiantes durante un paseo a un beneficio o plantación de café sin ningún otro propósito más que el de ir a degustar el producto y bajo el argumento de que ese tipo de actividades culturales «les gusta a los extranjeros». Esta situación pone al docente a merced de las exigencias y los horarios impuestos por la administración: no en pocas oportunidades se han visto casos de docentes que se encuentran con la sorpresa de que en su lista de obligaciones se ha programado un recorrido por los rápidos del Reventazón. Ahora bien, no se trata aquí de restarle importancia a las actividades recreativas y de descanso; sin embargo, los administradores con frecuencia las promocionan sin ninguna atención a su análisis cultural .

9 Philip Riley, Language Culture and Identity: An Ethnolinguistic Perspective (Londres: Conlinll um, 2(07) 176. 
Otro patrón relevante en torno a la lengua y a la cultura que desempeña un importante papel en la adquisición de la competencia cultural es la comunicación no verbal, puesto que, como señala McEntee 10, gran parte del significado de un mensaje se transmite mediante esta forma de comunicación. Si se considera que "un $65 \%$ de la población se comunica por medio de signos no verbales, [o sea] , la forma en que hablan, se mueven, gesticulan y manejan las relaciones espaciales» II, desconocer los códigos no verbales (los elementos kinésicos, proxémicos, cronémicos y paralingüísticos) de la cultura meta, precipita una infinidad de interpretaciones de significado y, por ende, malentendidos. Solo un acercamiento a las diferentes formas de expresión significativas de pensamiento y de estilo étnico, permitirá a los participantes interactuar en armonía dentro de la cultura meta.

4. ¿Cuáles valores? Dado que el análisis de los valores está sujeto al ideal de cada persona, conviene guiar la delimitación de los mismos en torno a la misión y visión del programa de estudios. En instituciones de sólida trayectorial2 no se debe olvidar que el quehacer académico debe ir de la mano con la misión y la visión institucional , y no supeditada a la opinión individual de sus participantes. De esta forma, los valores institucionales promoverán la renovación y el mejoramiento continuo y creativo de los procesos académicos y administrativos, con el fin de hacerlos más ágiles y eficientes, y en aras del fortalecimiento de la dimensión internacional de la vida académicaJ3 3

5. ¿Cuál identidad? Nuestra experiencia nos ha colocado frente a frente con el serna identidad - Ia relación que cada uno de nosotros mantiene consigo mismo-, que hemos descubierto intermitente

10 Eileen McEntee. Comunicación intercultural. Bases para la comunicación efectiva en el mundo actual (México: McGraw Hill . 1998) 382 .

11 McEntee, 384.

de la Universidad Nacional, Heredia, Costa Rica.

13 Universidad Nacional (UNA). El Modelo Pedagógico. Junio 
l'n el accionar de nuestra conciencia como comunidad académica. Si la identidad institucional dicta que se debe velar por: la formación de profesionales solidarios y comprometidos; el respeto a la diversidad en todas sus expresiones; el fomento a los procesos integrales, permanentes, contextualizados y propositivos; y el mejoramiento conti nuo de los procesos de gestión académica-administrativa14; queda en nuestras manos garantizar que, en el currículo para la enseñanza del ELE, haya concordancia entre la identidad de la lengua y la identidad de quien la habla para que no existan contextos o estrategias producto de una falta de homogeneidad discursiva interferente con el surgimiento de una nueva cultura.

\section{Valores y actitudes}

Para que la adquisición de una lengua sea efectiva, es necesario apropiarnos de su cultura; esto no es posible sin conocer el sistema de valores subyacente. Estos valores, según Rokeach, son «una creencia relativamente permanente en la que, para una situación particular, un modo específico de conducta es preferible personal o socialmente a otras formas de conducta»15. Para Vez, "el lenguaje es algo más que un instrumento PASIVO para la expresión de nuestras ideas; es nuestro mejor mecanismo para la formación de actitudes ante lo que nos rodea» 16 . Pero estas actitudes requieren alejarse de ciertos prejuicios producto de estereotipos o de categorización de los elementos individuales que opacan sus diferencias. Si bien cada uno de nosotros tiene una identidad social, pues formamos parte de una comunidad determinada, hemos de alcanzar un cambio de percepción social, y darle espacio a un aprendizaje cultural y a una eficaz comunicación interpersonal, de manera que asumamos la diversidad como un elemento enriquecedor y no como exduyente o discriminatorio.

14 UNA.

15 McEntee, 188.

16 José Manuel Vez, Formaci6n en Didáctica de las Lenguas Extranjeras (Santa Fe: HomoSapiens Ediciones, 2(01). 
La convivencia diaria y conjunta de estos estudiantes suele fomentar la categorización de los elementos individuales de la cultura de origen y, en alguna medida, subestimar la cultura meta. Por lo tanto, un componente esencial en el proceso de alejamiento de los prejuicios es la adecuada preparación, tanto de los anfitriones como de los aprendientes que viajan a otros países gracias a programas de intercambio con la única finalidad de aprender otra lengua. Mediante el uso eficaz de la lengua meta, el conocimiento de la cultura como mecanismo para la formación de actitudes y la oportuna interrela- ción con los participantes locales (por ejemplo, familias anfitrionas, tutores, personal administrativo, entre otros), los aprendientes logra- rán estructurar una identidad social en aras de la diversidad.

Utilidad del aprendizaje de una leftgua desde una perspectiva intercultural

En sus estudios, muchos expertos resaltan los beneficios que los aprendientes obtienen al adquirir una lengua dentro de esa perspectiva intercultural. En la presente reflexión, nos referiremos a los siguientes:

Conoci",knto de nuestra cultura

Conocer otra cultura supone explorar la nuestra, para reconocer semejanzas y diferencias. Esta etapa de introspección de uno mismo y de su medio, se presenta al enfrentarse a situaciones no experimentadas, entre las cuales podrían encontrarse desde un saludo, una reacción ante un problema de salud, hasta la forma de expresar el temor. No podremos lograrlo . sin haber desechado previamente los prejuicios que nos dificultan tener una visión real y amplia de esa cultura meta y de la nuestra; según McEntee, "el aprendizaje cultural se puede definir como un conjunto de situaciones intensas por las que el individuo llega a ser consciente de sí mismo y de otras personas, de tal manera que alcanza nuevos niveles de conciencia 
y de comprensión» 17. No sobra señalar lo imperioso que significa , para todo programa de ELE, facilitar y atender este elemento en la formación de los aprendientes, ya que la manera de interrelacionarse y, por ende, comunicarse, dependerá de ello: ¿ cómo nos juzgamos y cómo juzgamos a los demás?

Descubrimiento de las costumbres, tradiciones y valores de otras culturas

El conocimiento de la cultura meta, de la profunda y de la superficial, permitirá entender las costumbres o los gustos culinarios, estéticos y otros, y no solo de la cultura meta, sino también de otros miembros del programa. Tal descubrimiento es posible por medio de la observación. A quien aprende un idioma extranjero puede dársele una receta para actuar ante determinada situación o contexto, pero pronto se dará cuenta de que no todos reaccionan de la misma manera y que se requiere observar hasta el más mínimo detalle para encontrar la diferencia: ¿será la edad?, ¿ el color?, ¿ la hora?...

OrganiZQción de nuestros pensamientos y su comunicación con los demás

Una vez que nos conozcamos y hayamos recabado toda la información posible de la cultura meta, podremos organizar nuestras ideas y sentimientos, de tal forma que los expresemos desde el respeto a la diversidad y a los diferentes puntos de vista y, a su vez, los recibamos de igual forma. En otras palabras, habremos logrado reconocer, interpretar e incorporar estímulos contextuales para dar significado a los mensajes en una situación de comunicación en particular; de manera que logremos ser competentes en la cultura meta, después de haber pasado por las etapas de práctica y evaluación: Sí, era la edad.

17 McEntee, 66. 
Integración a la nueva sociedad con una actitud de tolerancia y respeto

Habrá que descartar el etnocentrismo, al creer que una cultura es mejor o peor que otra y apreciar la diversidad con una actitud de respeto por las diferencias culturales. El resultado de esta integra- ción intercultural será exitoso, en la medida en que los aprendientes (de cualquier nivel de competencia), tutores, profesores, anfitriones y otros, estén conscientes de que los estudios que vienen a realizar han de fundamentarse en la adquisición de esa dimensión intercultu- ral : ¿ Soy solo yo o también los demás?

Por lo tanto, les corresponde a los administradores proveer a los miembros de sus programas, a los aprendientes y a los homólogos de otras instituciones en el extranjero, del conocimiento sobre esos beneficios, y de las oportunidades y experiencias que les permitirán un verdadero aprendizaje intercultural : Mirá ..., si no era solo yo .

Materiales curriculares y metodologías de la clase

Si queremos que nuestros aprendientes logren un alto nivel de competencia cultural para estar en condiciones de afrontar los problemas de la dimensión social e intercultural, los materiales curriculares deben incluir las siguientes premisas:

a. Comunicaci ón es un proceso de negociación de significado y sus interpretaciones son plurales y variables.

b. Este proceso de negociación de significados está determinado por reglas discursivas de tipo social específicas de una lengua, las cuales son imprescindibles en la apropiación de la nueva lengua y su cultura. 
c. Estas reglas entran en juego por medio de procesos de interac- ción y, por ende, las estrategias de apropiación deberían en- marcarse en la dinámica de un contexto transaccional .

d. Como nos proponen Prado y otros18, el interculturalismo requiere que los grupos culturales adopten "posturas más abiertas de diálogo, interacción y mutuo reconocimiento de sus diferencias y diversidades como un hecho enriquecedor».

Quienes aprenden lengua extranjera necesitan desarrollar sensibilidad en cuanto a los prejuicios sociales y culturales que conllevan determinados actos de habla; una sensibilidad de este tipo resulta indispensable para comprender la nueva lengua como un medio de lograr la comunicación intercultural. Los materiales seleccionados o diseñados para este fin no deben limitarse a la utilización de actividades, herramientas audiovisuales o libros de texto que no se apeguen a nuestra cultura19. Recordemos que los materiales utilizados siempre estarán influidos por el contexto que les dio origen y éste por el sistema de valores que le subyace. Como consecuencia, la elección o diseño del material debe hacerse de una forma esmerada y permitir ese diálogo que tanto clama Prado.

Además, las metodologías de clase que incluyan giras o actividades de exposición cultural, han de someterse a estrictos controles pedagógicos, puesto que las actividades de recreación por realizar nunca han de sustituir el objetivo primordial del programa: el aprendizaje de la lengua desde una perspectiva intercultural .

18 Prado. 242.

19 Para un ejemplo de un libro de texßráapto el desarrollo de la destreza intercultural refiérase a Nuestro Mundo. Español como segundă lengua. Nivel \} (Herrera y Urbina, José: EUNED. 2009). 
Profesioftalizacióft de la coftpeteftcia ifttercultural de los actores del proceso

Para que los aprendices adquieran el esperado dominio de una lengua y una efectiva competencia cultural , los administradores de- ben real izar acciones inmediatas y eficaces, tales como:

1. Capacitación en materia cultural de todo el equipo involucrado en el proceso: Empezando por los docentes e incluyendo a las familias anfitrionas, y con mayor presencia de los directores y coordinadores de los programas, todos deben participar en una capacitación estructurada y efectiva acerca de:

a. ¿Qué es la cultura?

b. ¿Cuáles son los elementos característicos (profundos y superficiales) de la cultura costarricense y de las culturas de origen de nuestros estudiantes?

c. ¿Qué es un aprendizaje intercultural?

d. ¿En qué consiste el choque cultural y cuáles son sus etapas?

e. ¿Cómo actuar ante las diferencias de culturas de bajo o alto contexto?

f. ¿Cuál es la importancia de la comunicación no verbal?

2. Capacitación sobre resolución alternativa de conflictos y arbitraje internacional. Ante un problema intercultural, no se afecta únicamente la imagen individual de los involucrados, sino la imagen institucional y, dependiendo de la sensibilidad de losactores, la imagen global de la cultura meta. Más allá de visualizar las implicaciones, los administradores han de darse a la tarea de catalizar la realidad por medio de la reorganización y reconstrucción de la imagen de los programas. Para Rojas, en Mat020, muchas veces se

20 Daniel Mato, Diversidad cultural e interculturalidad en la educación superior. Experiencias en América Latina (Caracas: ASOCUN, 2008) 240.

94 
actúa bajo una premisa de «la cultura institucional» vista como: un conocimiento "científico» ya establecido en la comunidad académica que más bien se convierte en uno de los mayores obstáculos para el diálogo de saberes y la construcción colectiva de nuevas ofertas de formación.

3. Conformación de un cuerpo docente especializado. Además de los aspectos señalados en el apartado 1 , los docentes también deben tener un conocimiento muy claro de los distintos aspectos del español (fonético-fonológico, morfosintáctico , léxico) y, en especial , de las variedades utilizadas en Costa Rica. Además, unir este conocimiento a la historia, pues no es posible enseñar su cultura, si el docente mismo no sabe quién es quien. De allí la importancia de contar con un equipo docente especializado y experimentado en el ámbito intercultural; no basta con un grado académico, la experiencia intercultural (o, al menos su disposición a desarrollarla) resulta indispensable.

4. La inclusión de un protocolo del aprendizaje intercultural conjunto. El planeamiento horizontal y vertical debe abrir espacios para una perspectiva integradora en el currículo del programa y, en especial en las aulas, donde se aprecie cada cultura que conforma el grupo y, especialmente, un conocimiento de los aspectos importantes y necesarios para la vida cotidiana de la cultura costarricense y las prácticas propias de la cultura meta. Se han dado casos en donde las interpretaciones personalizadas de gestos y elementos proxémicos generan un descontrol en el desarrollo normal de una clase. Estas situaciones - algo tan simple como el uso de calzado informal o ropa deportiva en la clase de ELE - generan argumentaciones en torno a si un estudiante está irrespetando la autoridad, o si más bien la prenda se usa por razones de comodidad o por su bajo costo. 
¿Cuántos estudiantes habrán vuelto a casa sin la competencia intercultural esperada? Los aprendientes serán quienes han de reconocer la importancia de encontrarse en un entorno intercultural, donde sus actitudes o conductas van a impulsar el éxito o fracaso de esta experiencia y su disfrute. De allí la importancia de reconocer la influencia que ejercen los administradores en ese impulso. Si bien es cierto , la tarea no es fácil, no es el momento de aducir desconocimiento o, peor aún, desinterés; porque ya ha sido bastante difundida la necesidad de tomar cartas en el asunto y enriquecer nuestros programas a favor de los miles de estudiantes que llegan a nuestro país con el deseo de aprender lalengua.

¿Estamos frente a una interpretación vulgar? La práctica etimológica ha interpretado nuestros esfuerzos de convertimos en una comunidad académica crítica y los ha reducido a simples teorías y ciencias sostenidas por fuerzas históricas . La interpretación administrativa no debe ser unilateral , ni basada en simples juicios subjetivos. Porque, de ser así, restringiremos la educación a la simple transmisión de información y no a un proceso de investigación-acción que desarrolle las aptitudes y destrezas de cambio cultural de sus participantes.

¿Hemos deformado acaso los significantes? Luci021 advierte que pedagogía responde científicamente a la pregunta ¿cómo edu- car? y didáctica lo hace con la pregunta ¿cómo enseñar? En manos de los administradores está transportar la visión didáctica de un pro- grama de ELE a un momento pedagógico, a un proceso reflexivo de observación y planeamiento, en que el aprendizaje deje de ser una conducta intermitente .

Reflexiones onomasiológicas... con respuestas semasiológicas, sin duda alguna.

21 Ricardo Lucio, "Educación y pedagogía; enseñanza y didáctica: Diferencias y relaciones», Revista de la Universidad de La Salle (Madrid) 17, 1998. 